\title{
Editorial Spread sexual medicine knowledge through the written word: IJIRjsm
}

\author{
International Journal of Impotence Research (2003) 15, 79. doi:10.1038/sj.ijir.3901003
}

Since health is a fundamental human right, so must sexual health also be a basic human right (World Health Organization). Men and women who have personal distress (bother, fear, humiliation, loss of self-esteem, anger, anxiety, loss of relationship, etc) from sexual health problems need safe and holistic (psychologic and biologic) environments in which to seek evidence-based management for their sexual concerns. Men and women with health concerns expect and receive this standard of health care for all other nonsexual health issues and should expect to receive no less for their sexual health inquiries.

Sexual medicine is the branch of medicine that embraces the study, diagnosis and treatment of men and women with sexual health concerns. Health professionals (therapists, physicians and health-care clinicians), who practice sexual medicine on patients with various sexual health conditions need up-to-date scientific research concerning the basic knowledge and mechanisms of sexual physiology and the pathophysiology of sexual health concerns. As sexual function is intimately related to mind, body and relationship issues, sexual medicine practitioners must work together to help patients. Therapies need to be defined, safe, effective and based when possible on multi-institutional placebocontrolled, double-blind studies.

The International Journal of Sexual Medicine: The Journal of Sexual Medicine is a resource for all therapists, physicians, health-care clinicians and researchers interested in sexual medicine whether it be basic science research, diagnosis and treatment or clinical trials of new medications. Evidence-based medicine is incontrovertible. The journal will publish science so that those interested and involved in the field may learn and progress. There has been science for sexual dysfunction for men for 30 years. In response, there has been progress in researching and developing therapies for women with sexual dysfunction, hence the new name to this journal. The scope of the IJIRjsm has been broadened to encompass the whole field of sexual medicine and all the disciplines involved.

Based on numerous epidemiologic studies performed all over the world, there exist millions of men and women with persistent complaints about their sexual function that cause them personal distress. Too many nonsexual medicine physicians are afraid to inquire about the status of their patients' sexual lives. As a result, many men and women patients do not even receive fundamental psychologic or medical care for their sexual problems. If patients are brave enough to announce their sexual issues, they should not be distracted by an academic community that denies the very existence of sexual health problems and claims quite erroneously that sexual dysfunctions are an invention of the financially motivated. As long as there exist patients distressed about a health problem, there must be researchers seeking to discover why these problems exist, and therapists, physicians and health-care clinicians seeking to treat these patients with evidence-based safe and effective therapies.

In sexual medicine, the unfortunate reality is that there is a paucity of government funds for sexual medicine investigations. Thought leaders are often only able to perform research utilizing nongovernment funding. As in all of medicine, research needs to be conducted in a moral and responsible fashion independent of government or nongovernment funding sources.

In the end, the sexual medicine psychologic and biologic community needs to have multidisciplinary, academic and scientific organizations to provide opportunities for communication among scholars, researchers and practitioners about men and women's sexual function, to support the highest standards of ethics and professionalism in research, education and clinical practice of men and women's sexual health, and to provide the public with accurate information about men and women's sexual health.

Through the written word in the IJIRjsm and other sexual medicine journals, knowledge concerning the field of sexual medicine will be spread. In the end, it is ONLY through the written word that we can fulfill our mission as health-care practitioners of 'helping others'. Restoring sexual function in a man or woman with sexual dysfunction is extremely rewarding. Please help progress in the field of sexual medicine through your manuscripts, which will be published in the leading journal on sexual medicine.

Irwin Goldstein 